
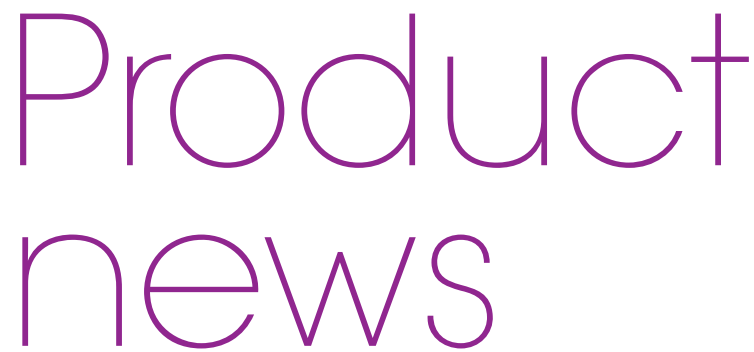

Product news is provided as a service to readers using text and images from the manufacturer, supplier or distributor and does not imply endorsement by BDJ Team. Normal and prudent research should be exercised before purchase or use of any product mentioned.

\section{SMART TOOTHBRUSH WILL PROVIDE REAL TIME GUIDANCE TO PATIENTS}

On 1 November 2017 at an exclusive event in Central London, a revolutionary new dental product was launched to tackle Britain's poor toothbrushing habits. Brushlink is a device that costs less than $£ 30$ that will transform any toothbrush into a smart toothbrush.

Brushlink combines a tracking device attached to your toothbrush with a smartphone app. The smartphone app provides the user with real time brushing guidance and performance monitoring. Each brushing session is scored and monitored and the cumulative data can be shared with dental professionals so that they can provide targeted help and advice to the patient.

The tracking device is a tiny hi-tech Bluetooth unit that attaches easily to any toothbrush and monitors brushing frequency, duration and, for the first time, angulation, so that correct brushing angles can be encouraged, reinforced and monitored to help prevent gum disease.

Brushlink was founded by CEO Dr Dev

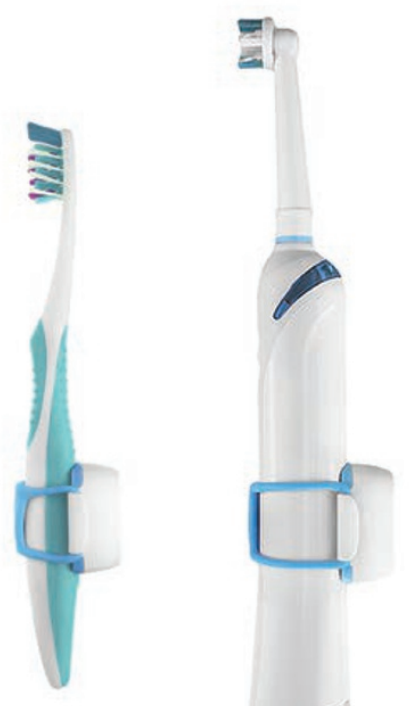

Patel (featured in the $B D J$ in November: http://go.nature.com/2hoKg8X).

Through using Brushlink, patients can sync their brushing data with a Brushlink certified dental practice, for tailored dental hygiene recommendations, and earn bespoke discounts.

For more information about Brushlink and to order, visit https://brushlink.com/.

\title{
CLEAN AND UNCOMPLICATED
}

Dental Express can help you comply with infection control protocols with ease, with the Gojo Foam Soap Dispenser.

The large $700 \mathrm{ml}$ cartridge will save you time on repeat refills. Available in either chrome and black or grey and white, you can choose the best fit for your practice. Touch-free, thus reducing the chance for cross-contamination and reducing mess, the Gojo Foam Soap Dispenser is easy to use, and can be refilled within seconds. The dispenser is free on loan when you buy the compatible cartridges, so you can find out how well it fits within your practice with a low initial outlay.

For more about the Gojo Foam Soap Dispenser, visit www.dental-express.co.uk or call 08007076212 .
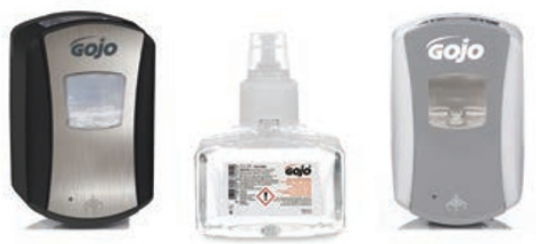
THE HUMAN HAND IS
REMARKABLE
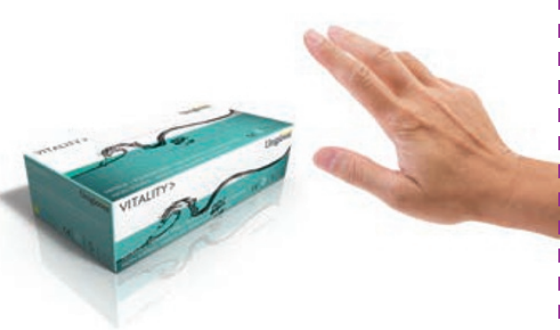

The human hands are amazing instruments of touch, expression and ability. They are probably the most valuable tools in your armamentarium and they should be looked after properly with high quality gloves from the Unigloves Vitality range.

Unigloves understands that you need premium quality and strength for effective protection but as experts in the field, Unigloves also know how to care for your hands properly.

Vitality gloves are available in a variety of sizes for the most suitable and comfortable fit. They feature doublechlorinated beaded cuffs making them easy to put on and remove as well as a micro-roughened texture to ensure optimal grip.

For an added bonus, Unigloves also coat the internal surface of each glove with a soothing Vitamin E (Lano-E) moisturiser to keep your hands flexible and hydrated.

The Vitality range includes:

- Vitality Nitrile - white, powder-free with Lano-E coating

- Vitality Latex - white, powder-free with Lano-E coating

- Vitality Latex scented - green, powder-free with Lano-E coating and scented with a pleasant citrus and peppermint flavour.

Your hands are remarkable and for gloves that offer protection and care, contact Unigloves today.

For further information about Unigloves products, visit www.unigloves. co.uk.

If you would like to promote your products or services direct to the dental industry in BDJ Team, call Andy May on 02078434785 or emaila.may@nature.com 